\title{
A New Fault Diagnosis Model for Circuits in Railway Vehicle Based on the Principal Component Analysis and the Belief Rule Base
}

\author{
Hao Wu, ${ }^{1}$ Bangcheng Zhang, ${ }^{2}$ Zhi Gao $\mathbb{D}^{3,4}$ Siyu Chen, ${ }^{2}$ and Qianying $\mathrm{Bu}^{5}$ \\ ${ }^{1}$ China Oil and Foodstuffs Corporation, Information Management Center, Beijing 100020, China \\ ${ }^{2}$ School of Mechanical and Electrical Engineering, Changchun University of Technology, Changchun 130012, China \\ ${ }^{3}$ School of Mechatronic Engineering, Changchun University of Technology, Changchun 130012, China \\ ${ }^{4}$ School of Mechanical and Electrical Engineering, Changchun University of Science and Technology, Changchun 130022, China \\ ${ }^{5}$ Changchun Fuwei an Daotuo Automobile Trim System Co. Ltd., Changchun 130033, China
}

Correspondence should be addressed to Zhi Gao; gaozhi@ccut.edu.cn

Received 11 August 2021; Revised 27 October 2021; Accepted 11 November 2021; Published 28 December 2021

Academic Editor: Chuan Li

Copyright ( $\odot 2021$ Hao Wu et al. This is an open access article distributed under the Creative Commons Attribution License, which permits unrestricted use, distribution, and reproduction in any medium, provided the original work is properly cited.

\begin{abstract}
Circuits are considered an important part of railway vehicles, and circuit fault diagnosis in the railway vehicle is also a research hotspot. In view of the nonlinearity and diversity of track circuit components, as well as the diversity and similarity of fault phenomena, in this paper, a new fault diagnosis model for circuits based on the principal component analysis (PCA) and the belief rule base (BRB) is proposed, which overcomes the shortcomings of the circuit fault diagnosis method based on data, model, and knowledge. In the proposed model, to simplify the model and improve the accuracy, PCA is used to reduce the dimension of the key fault features, and varimax rotation is used to deduce the fault features. BRB is used to combine qualitative knowledge and quantitative data effectively, and evidential reasoning (ER) algorithm is used to carry out the inference of knowledge. The initial parameters of the model are optimized, and the optimal precondition attributes, rule weights, and belief degree parameters are obtained to improve the accuracy. Through the training and testing of the model, the experimental results show that the method can accurately diagnose the fault of the driver controller potentiometer in the railway vehicle. Compared with other methods, the model shows high accuracy.
\end{abstract}

\section{Introduction}

The circuits are important parts of the railway vehicle [1] Under the adverse working conditions of high temperature and vibration environment of the railway vehicle, the failure probability is very high, and the diagnosis is difficult. Any damage to the circuits of a railway vehicle may result in security issues. In order to enhance the security of railway vehicle, the circuit fault diagnosis of railway vehicles is a very important way to find the fault in time. However, the development of circuit fault diagnosis is slow; due to the complexity and nonlinearity of the electronic system, the tolerance of the components, and the diversity of the faults, even some of the faults are slowly changed, and whether the circuit is faulty cannot be determined unilaterally [2].

Based on the particularity of the circuit system, it can be known that circuit failure is not a failure mode in the traditional sense. It is necessary to timely feed back the failure and future failure information to the driver. Therefore, the fault detection method cannot use the traditional fault detection method. It is necessary to develop a suitable fault diagnosis method according to the characteristics of the electronic system.

At present, fault diagnosis methods are mainly divided into two categories: quantitative methods and qualitative methods. Quantitative methods are mainly based on 
data-driven methods, using large amounts of data to train the system, which are accurate. However, they cannot add expert knowledge and output the fault samples corresponding to the fault category label, which belong to the black box and cannot output other diagnostic information, such as the probability of the fault sample belonging to each fault category. There are mainly backpropagation neural network (BPNN) [3], support vector machine (SVM) [4], and DS evidence theory fusion method [5]. Qualitative methods can build a knowledge base or use the empirical knowledge of experts. The more the knowledge base, the higher the diagnostic accuracy, but the data cannot be used for learning and updating. For example, Huang et al. [6] proposed an improved hidden Markov model for rail transit motor faults. Li [7] proposed an expert system-based fault diagnosis method for locomotive electric drive circuits. Yang et al. and Zhang et al. [8,9] proposed reasoning methods based on DS theory, decision theory, and rule base. This method adds rule parameters on the basis of traditional rules and establishes the learning model of the RIMER expert system.

Combining qualitative and quantitative methods can effectively solve the problem of fault classification and use data for learning and updating. Liu and Tong and Zhang $[10,11]$ put forward the method of combining neural network and expert system to study the fault of locomotive wheel pair, which effectively solves the misdiagnosis rate and real-time problems in traditional faults. However, the problem of the probability of failure has not been resolved. Therefore, this paper proposes a new PCA-BRB fault diagnosis model. At first, when the dimensionality of the data was too high, PCA was used to reduce the dimensionality of the feature quantity. In order to extract more representative and physically meaningful fault feature quantities, the factor rotation method, that is, the maximum variance orthogonal rotation, is used to reduce the dimension of the load matrix through factor analysis to obtain the physical meaning of the fault feature quantities. Then, the reasoning method of the belief rule base using the evidence reasoning method (RIMER) is used to diagnose the fault. The combination of principal component analysis and factor analysis can effectively reduce the number of input confidence rules in the confidence rule base (BRB) and improve learning efficiency and fault diagnosis accuracy. This paper mainly solves the problems of fault in railway vehicle circuits. The RIMER expert system can be used to classify the faults effectively; meanwhile, calculated sample belongs to the probability of each failure categories. Therefore, it can be utilized for the circuits of engineering practice. The main work in this paper includes the following:

(1) Due to the high complexity of the rail vehicle line model, the variety of data, and the difficulty of data monitoring, it is easy to cause a combination explosion, especially in a large-scale system, and it is difficult to monitor a large number of variables in each subsystem. This results in a need for fault diagnosis methods that can work with a limited set of monitoring signals. In order to simplify the model and improve the accuracy of using principal component analysis (PCA) to construct the factor model, [12], use maximum variance rotation to invert the relationship between principal components and feature quantities, and select key fault features through dimensionality reduction.

(2) In order to classify the fault samples of the circuits of rail vehicles, the RIMER method based on evidential reasoning is used to diagnose the fault. Knowledge is expressed by BRB and reasoned by ER. The input is a prerequisite attribute sample after dimensionality reduction, the basic probability mass of each confidence rule is established, and then the rules are combined by ER, and the output is the probability of each fault; according to the corresponding fault probability and the fault type semantic value, the fault type semantic value of the final output is calculated.

(3) Fmincon's active-set algorithm is employed as the optimization method to train and optimize BRB parameters. The objective function is the minimum variance of the real fault semantic value and the initial BRB output. As a result, the optimized BRB can reflect system's behavior accurately.

(4) The new PCA-BRB-based model was proposed and applied to an actual engineering system to verify the validity of the new model. Compared with the other approaches, the proposed model has shown higher accuracy.

The rest of this paper is organized as follows. In Section 2 , a new fault diagnosis model for circuits in railway vehicle based on the principal component analysis and the belief rule base is proposed. In Section 3, fault diagnosis steps are put forward. In Section 4, the potentiometer is chosen as a numerical example to validate the efficiency of the new proposed model. In Section 5, conclusions are provided.

\section{Fault Diagnosis Theory Based on PCA-BRB}

2.1. Feature Dimension Reduction Method for PCA. The railway vehicle circuits have many electronic components and many kinds of fault characteristics, and the characteristics of faults are complicated, but it is difficult to monitor a large number of variables. It is especially important to effectively reduce the number of features and find smaller dimensions and more representative features without changing the qualitative knowledge contained in the data itself and to simplify the fault diagnosis model at the same time.

Principal component analysis (PCA) is an effective method for statistical analysis of data, which is based on the Karhunen-Loeve decomposition. Its purpose is to find a set of vectors in the data space to explain the variance of the data as much as possible. Through a special vector matrix, the data are mapped from the original high-dimensional space to the low-dimensional vector space. After the dimensionality is reduced, the vector retains the main information of the original data, making the data easier to process [13]. But 
the result of PCA is to obtain the principal component which contains the comprehensive index. The main component is reconstructed, and it will lose some qualitative knowledge and does not have the actual physical meaning, which is not conducive to the construction of the BRB model. The factor analysis, through the study of the internal relationship between the correlation matrix and covariance matrix of many variables, finds out a few random variables (factors) that can integrate the main information of all variables.

Therefore, a method of combining principal component analysis with varimax rotation is proposed to reduce the dimension of fault feature. Firstly, PCA is used to extract the principal component, and then the principal component is used to carry out the reverse reasoning [14], using the method of maximum variance rotation in the factor analysis method to solve the load matrix. The purpose is to make the absolute value or square value of each element of the load matrix as much as possible two-level differentiation, that is, the absolute value or square value of a few elements is as large as possible, while other elements close to zero. The fault characteristic quantity with the absolute value of more than 0.9 is selected as the key fault characteristic of the principal component. Specific steps are as follows.

2.1.1. Standardization of Raw Data. The original dataset of the fault characteristic value of electronic circuits is $\left(x_{i j}\right)^{T \times P}$ (where $T$ is the number of samples and $P$ is the number of fault features). In order to eliminate the influence of the different dimensions of original variables and large numerical difference, it is necessary to standardize the original variables, that is:

$$
x_{i j}^{*}=\frac{x_{i j}-x_{j}}{\sqrt{S_{i j}}}, \quad i=1,2, \ldots T ; j=1,2, \ldots p,
$$

where

$$
\begin{aligned}
& x_{j}=\frac{1}{T} \sum_{i=1}^{T} x_{i j}, \\
& S_{i j}=\frac{1}{T-1} \sum_{i=1}^{T}\left(x_{i j}-x_{j}\right)^{2} .
\end{aligned}
$$

2.1.2. Establishing Correlation Matrix and Calculating the Matrix Eigenvalue and Eigenvector

$$
R=\frac{1}{T-1} X^{*} X^{T},
$$

where $X^{*}$ is data matrix of standardization. Eigenvalue $\lambda_{j}$ and eigenvector $e_{j}$ are calculated by $R$, for $j=1,2, \ldots p$.

2.1.3. Selection of Principal Component. The contribution rate of the $j$ th principal component to the total variance is calculated, that is, the variance contribution rate is calculated:

$$
\text { per }=\frac{\lambda_{j}}{\sum_{j=1}^{P} \lambda_{j} \times 100 \%} .
$$

The $P$ principal components are sorted from large to small order according to the contribution rate; usually, the principal component number $n$ whose cumulative contribution rate is greater than $95 \%$ is selected.

\subsubsection{Establishing a Factor Model}

$$
y_{n}=E x_{p} .
$$

In general, the PCA transforms $P$ vectors $\left(x_{1}, x_{2}\right.$, $\left.x_{3}, \ldots x_{p}\right)$ to $n$ vectors $\left(y_{1}, y_{2}, y_{3}, \ldots y_{n}\right)$. where $E$ represent the load matrix, $E=\operatorname{dig} \sqrt{\lambda_{\mathrm{n}}} e_{n p}$.

\subsubsection{Orthogonal Rotation of Load Matrix}

$$
\begin{aligned}
B & =E Q \\
& =\left(b_{i j}\right) .
\end{aligned}
$$

$\mathrm{B}$ is the rotation factor load matrix. Let matrix B's variance of sum be max, and thus orthogonal matrices $Q$ and $B$ are obtained. The critical fault characteristics of $b_{i j}>0.9$ are selected.

Through principal component analysis, the input of the expert system of the rule base is reduced, and the number of rules and the structure of the model are simplified, which can greatly improve the learning speed.

2.2. RIMER Method. The belief rule base inference methodology using the evidential reasoning approach (RIMER) is mainly composed of two parts, which are the regular representation of known information and inference of decision process rules. At present, this method is effective in solving classification problems, pattern recognition, fault diagnosis, and so on. Because of the nonlinear characteristics of the circuits, the diversity of the components, and the complex circuit connection, the mathematical model is difficult to be established when the fault of an electronic component or a branch is not proportional to the total output. However, the rule base expert system does not need to establish mathematical model through historical data and expert knowledge. It is only necessary to carry out the knowledge representation by means of the rule base, and the inference of knowledge is realized by ER inference. It belongs to white box diagnosis, which has clear observation of the reasoning process and a clear explanation of the diagnostic results. This paper presents a method of fault diagnosis for railway vehicle electronic circuits based on the belief rule base inference methodology using the evidential reasoning approach (RIMER).

2.2.1. The Knowledge Representation of the BRB. Its main purpose is to calculate the circuit input data and the rules of the rule base operations. After regularization, the known input contains the fault information of each rule, and the 
fault information is an equivalent to known input weights assigned to each rule. The regularization process is not a simple IF-THEN rule, and it is a more comprehensive introduction of fault feature weight and rule weight. Sometimes, the known data are highly similar to a rule. But this rule is not important, or the premise attribute is not important, and the weight after regularization is not necessarily very high [15]. The $k$ th rule of the belief rule base is represented as

$$
\begin{aligned}
& \text { If } x_{1} \text { is } A_{1}^{k} \wedge x_{2} \text { is } A_{2}^{k} \text { is } A_{M}^{k}, \\
& \text { Then }\left\{\left(D_{1}, \beta_{1, k}\right), \ldots,\left(D_{N}, \beta_{N, K}\right)\right\} . \\
& \text { With a rule weight } \theta_{k} \text { and } \\
& \text { fault feature weight } \delta_{1, k}, \ldots, \delta_{M_{k}, k} \text {, }
\end{aligned}
$$

where $X$ is input vector; $A^{k}$ represents a collection of entered reference values by the rule $k, A^{k}=\left\{A_{1}^{k}, A_{2}^{k}, \ldots, A_{M}^{k}\right\}$, $k=1,2, \ldots, L$, where $L$ is the number of all rules; $D$ is the result vector, and $D=\left[D_{0}, D_{1}, D_{2}, \ldots, D_{N}\right]$, where $N$ is the system states number including the normal state; $\beta^{k}$ is the vector belief degree, which is the possibility that the result of fault diagnosis results in various states; $\theta^{k}$ is the rule weight, which represents the importance of the corresponding rules; and $\delta_{j}$ is the weight of fault feature, which indicates the importance of the $j$ th precondition (fault feature).

The BRB expert system can effectively use various types of information, and a nonlinear model is established by the rail vehicle electronic circuits characteristic input and fault class output. Compared with the traditional IF-THEN rules, it provides a way to contain more information, more close to the actual knowledge expression.

2.2.2. ER Algorithm. The BRB is the expression of frame of knowledge, but after a group of fault feature input, how to combine the $L$ rules in the rule base is important, so that the results can be achieved in the fault detection and diagnosis. Specific steps have to be achieved by reasoning algorithm. The disadvantage of the traditional Dempster evidence theory is that there is no fusion of conflict events [16]. To solve this problem, there are two kinds of solutions, one is that the Dempster rule is flawed and needs to be modified; the other is that it is not related to the Dempster rule, and the reason lies in the modelling not being accurate, and it needs to preprocess the evidence and then make a combination [17]. However, most of the modifications to the rules have destroyed the combination rule of the Dempster rules, and the pretreatment of the evidence changed the specificity of the evidence and did not take into account the reliability and importance of the evidence. Li et al. [18] put forward a new evidence combination rule of evidence reasoning (ER) algorithm; after preprocessing the evidence, the Dempster rule is used to synthesize the algorithm, which not only satisfies the commutative law and associative law but also keeps the specificity of the evidence in the preprocessing. Specific steps are as follows [15]:

(1) Calculating the activation weights of belief rules: if $x_{i}$ is assumed to be the $i$ th input, the activation weights in the $k$ th rule can be expressed as

$$
\begin{aligned}
& \omega_{k}=\frac{\theta_{k} \prod_{i=1}^{M}\left(\alpha_{i}^{k}\right)^{\bar{\delta}_{i}}}{\sum_{l=1}^{L} \theta_{l} \prod_{i=1}^{M}\left(\alpha_{i}^{k}\right)^{\bar{\delta}_{i}}}, \\
& \omega_{k}=\theta_{k} \prod_{i=1}^{M}\left(\alpha_{i}^{k}\right)^{\bar{\delta}_{i}}
\end{aligned}
$$

In this paper, two methods for calculating RIMER activation weights of circuit fault diagnosis are put forward. The activation weights in equation (9) are required to be normalized, that is, $\sum \omega_{k}=1$; equation (10) is nonnormalization; when there are many rules of circuit belief rules, the normalized weight will result in the small weight of all rules, so that the fusion result is very close to the mean value. The equation (10) is used to calculate when the conflicts between the rules are not very large or the weight of the conflicting evidence is small. Fusion after normalization is used when there is a large conflict in the rules and the weight of conflict evidence is large.

$$
\alpha_{i j}\left(x_{i}^{*}\right)= \begin{cases}\frac{x_{i(k+1)}-x_{i}^{*}}{x_{i(k+1)}-x_{i k}}, & j=k\left(x_{i k} \leq x_{i} \leq x_{i(k+1)}\right), \\ \frac{x_{i}^{*}-x_{i k}}{x_{i(k+1)}-x_{i k}}, & j=k+1, \\ 0, & j=1,2, \ldots\left|x_{i}\right|, j \neq k, k+1,\end{cases}
$$


where $\omega_{k} \in[0,1], \quad k=1,2, \ldots, L ; \quad a_{i, j} \quad$ is the matching degree of the input information which is based on rules or utility. The input information is the matching of $i$ attribute of $j$ rules.

(2) ER algorithm: the final output of BRB $S(x)$ can be realized by the combination of all the rules in the ER algorithm:

$$
S(x)=\left\{\left(D_{n}, \widehat{\beta}_{n}\right)\right\}, n=1, \cdots, N .
$$

The confidence level of $D_{n}$ is shown in equation (8):

$$
\begin{aligned}
& \widehat{\beta}_{j}=\frac{\mu \times\left[\prod_{k=1}^{L}\left(\omega_{k} \beta_{n, k}+1-\omega_{k} \sum_{n=1}^{N} \beta_{n, k}\right)-\prod_{k=1}^{L}\left(1-\omega_{k} \sum_{n=1}^{N} \beta_{n, k}\right)\right]}{1-\mu \times\left[\prod_{k=1}^{L}\left(1-\omega_{k}\right)\right],} \\
& \mu=\left[\sum_{n=1}^{N} \prod_{k=1}^{L}\left(\omega_{k} \beta_{n, k}+1-\omega_{k} \sum_{n=1}^{N} \beta_{n, k}\right)-(N-1) \prod_{k=1}^{L}\left(1-\omega_{k} \sum_{n=1}^{N} \beta_{n, k}\right)\right]^{-1} \cdot \\
& \text { and (14) can be obtained by the }=\frac{1}{T} \sum_{i=1}^{T}\left(y_{i}-y_{i r}\right)^{2},
\end{aligned}
$$
activation weight equation (9) or (10). Therefore, it can be seen that $\hat{\beta}_{n}$ is described by the function of confidence $\beta_{n, k}(n=1, \ldots, N, k=1, \ldots, L)$, rule weight $\theta_{k}(k=1, \ldots$, $L)$, and fault feature weight $\delta_{j}$.

Finally, the discriminant result of output can be expressed as

$$
y_{i}=\sum_{1}^{N} D_{n} \beta_{n}, \quad n=1,2, \ldots, N .
$$

2.2.3. Optimization of the BRB Parameters. The initial BRB parameters are usually given by experts based on prior knowledge and historical information. It is difficult to determine the precise values of these parameters. Especially in the rail passenger car driver's console, there are many types of faults and the diagnosis accuracy is required. In the case of high demand, the driver's console fault diagnosis result output by the initial BRB will deviate from the real result. The accuracy of the evaluation is reduced. Therefore, in order to improve the evaluation accuracy, the initial BRB needs to be optimized, and the purpose of optimization is to minimize and optimize the error between the actual output result and the initial $\mathrm{BRB}$ output result. So, it is necessary to optimize the initial BRB, and fmincon's active-set algorithm is used in this paper [19].

The BRB parameter vector is $V=\left[\theta^{1} \cdots \theta^{K} \cdots \delta^{1} \cdots\right.$ $\left.\delta^{m} \cdots \beta_{1}^{k} \cdots \beta_{n}^{k}\right]^{T}$, and its constraints are

$$
\begin{aligned}
& \quad \min \operatorname{MSE}\left(y\left(\theta^{k} \delta^{m}, \beta_{n}^{k}\right)\right), \\
& 0 \leq \theta_{k} \leq 1, \\
& 0 \leq \beta_{n}^{k} \leq 1, \\
& \sum_{n=1}^{N} \beta_{n}^{k}, \quad k=1,2, \ldots L .
\end{aligned}
$$

The objective function [20] is where $y_{i r}$ is the real referenced value of the data.

\section{PCA-BRB Fault Diagnosis Model for Circuits in Railway Vehicle}

3.1. Circuit Fault Diagnosis Flowchart. The flowchart of the PCA-BRB fault diagnosis model for circuits is shown in Figure 1.

3.2. Circuit Fault Diagnosis Procedure. The steps of the PCABRB fault diagnosis model for circuits are shown in Table 1.

\section{Case Study}

The driver controller is the key electrical equipment on the rail vehicle, and it is the main control device used by the driver to operate the locomotive. All instructions for the traction, braking, and speed regulation of the motor train unit are realized by the driver controller. It mainly uses the low-voltage electrical apparatus of the control circuit to indirectly control the electrical equipment of the main circuit; whether the action is well will directly affect the smooth operation of the locomotive and the realization of various conditions. The small fault of the driver controller will bring a lot of security risks to the locomotive, for example, "motor vehicle suddenly channels endplay," no voltage and no electricity, the electronic circuit receives the wrong instruction to produce the disoperation, and so on, and these will bring very big threats to the equipment safety and the driving safety. So, using numerical observations accurately to diagnose for hidden failures is particularly important.

The driver controller is prone to electrical failure, and the potentiometer circuit fault of it directly leads to serious problems of the locomotive, for example, the internal open circuit of the potentiometer could lead to no voltage and no electricity of the railway vehicle, element parameters change, and the external load of the potentiometer affects 
TABLE 1: PCA-BRB fault diagnosis model for circuits.

Step 1: fault feature selection by the PCA method.

Step 1.1: constructing the standardized data $x_{i j}^{*}$ by equations (1)-(3).

Step 1.2: calculating the correlation matrix $R$ by equation (4) and calculating the eigenvalue $\lambda_{j}$ and characteristic vector $e_{i}$ of $R$ matrix.

Step 1.3: calculating the principal component of eigenvalue by equations (6) and (7).

Step 2: the establishment of the initial BRB.

Step 2.1: defining semantic values of system input and output.

Step 2.2: setting the initial parameters of the BRB.

Step 2.3: calculating the initial BRB output semantic value by equations (8)-(14).

Step 3: optimization of the BRB parameters.

Step 3.1: calculating the initial BRB and real output variance MSE, as the target function by equation (20).

Step 3.2: establishing the constraint by equations (16)-(19).

Step 3.3: selecting the best parameters in the current generation.

Step 4: electronic circuit fault diagnosis.

Step 4.1: the testing data are transformed to the belief degrees by equation (11).

Step 4.2: calculating the activated weights of rules in the optimized BRB by equation (9) or (10).

Step 4.3: deriving the distributed output of the system's state by equations (13) and (14).

Step 4.4: calculating the expected utility of the fault diagnosis by equation (15).

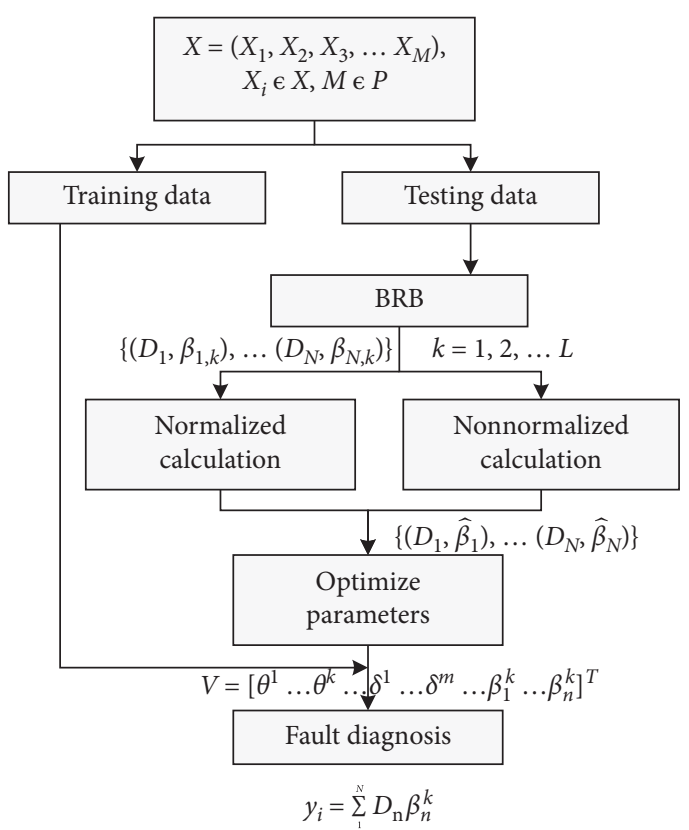

FIgURE 1: The flowchart of the PCA-BRB fault diagnosis model for circuits.

the voltage value of the traction system's acquisition accuracy. The purpose of this section is to solve the problem of fault diagnosis of the driver controller and take a large number of fault diagnosis problems in the circuit of the potentiometer as an example. The method is used to establish the rule base of the driver controller, and the corresponding learning model is established. The PCA is used to select the fault feature, so as to simplify the learning model. In order to verify the proposed fault diagnosis model for rail vehicles, the most common faults in working process of the driver controller potentiometer are diagnosed, and these five states are normal work, the forward bias of the component parameters, the negative deviation of the component parameters, the external load, and internal open circuit.
4.1. Experimental Design. In this paper, the fault diagnosis of the implementation of the framework is from the literature [21], as shown in Figure 2. The framework is divided into 5 stages: fault circuit generation, fault data simulation, fault feature extraction, fault diagnosis, and parameter optimization $[22,23]$.

\subsubsection{Generating the Potentiometer Fault Circuit.} Potentiometer faults can be divided into hard and soft faults. Hard faults, such as open circuit or short circuit, will cause the topology change of the potentiometer circuit, and the circuit function will change greatly.

The soft fault is the actual value of the component parameter beyond the tolerance range, leading to the reduction of the working performance of the potentiometer, the speed command error, and so on.

In the generation phase of the potentiometer fault circuit, a variety of faults are injected into the potentiometer simulation circuit by the mutation operation, and a series of circuit under test (CUT) variants with different fault modes are obtained. Fault generation is shown in Table 2. Among them, the forward bias of the component parameters and the negative deviation of the component parameters are the PCH mutation operations, and they are uniformly distributed $\left(U\left[0.1 X_{n}, X_{n}-2 t\right]\right.$ and $\left.U\left[X_{n}+2 t, 2 X_{n}\right]\right)\left(X_{N}\right.$ is the nominal value of component and $t$ is tolerance value of the component); potentiometer external load fault is GRB mutation operation which is uniform distribution $(U[10 \Omega, 1 K \Omega])$; potentiometer internal open circuit is ROP mutation operation which is uniform distribution $(U[100 k \Omega, 100 M \Omega])$.

In this paper, potentiometer schematic diagram is adapted from the literature [23]. Figure 3 shows the simulation of the driver controller potentiometer in Proteus. Train management system (TMS) provides $15 \mathrm{~V}$ analog power supply for the controller, where $R V 1$ and $R V 2$ are "traction" and "brake" sliding rheostats. When the speed control handle A of the master controller is manipulated and rotated in the $R V 1$ or $R V 2$ area, the output voltage of the 


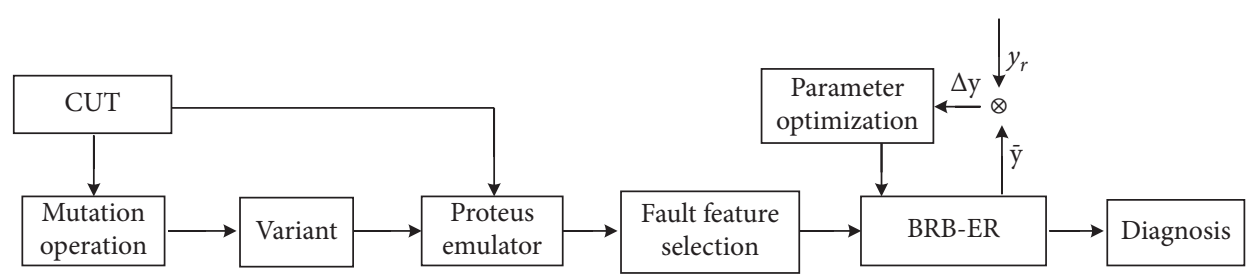

FIgUre 2: The structure of the PCA-BRB model.

TABLE 2: Mutation operators.

\begin{tabular}{|c|c|c|}
\hline Operators & Name & Description \\
\hline $\mathrm{PCH}$ & Parameter changes & Specified parameters of the component diverge the tolerance range \\
\hline GRB & $\begin{array}{l}\text { Global resistive } \\
\text { bridging }\end{array}$ & A resistor with a very low resistance is connected between the two nodes of different components \\
\hline ROP & Resistance open & $\begin{array}{c}\text { A resistor with a high resistance is connected between the ports of the component to represent the } \\
\text { open circuit }\end{array}$ \\
\hline
\end{tabular}

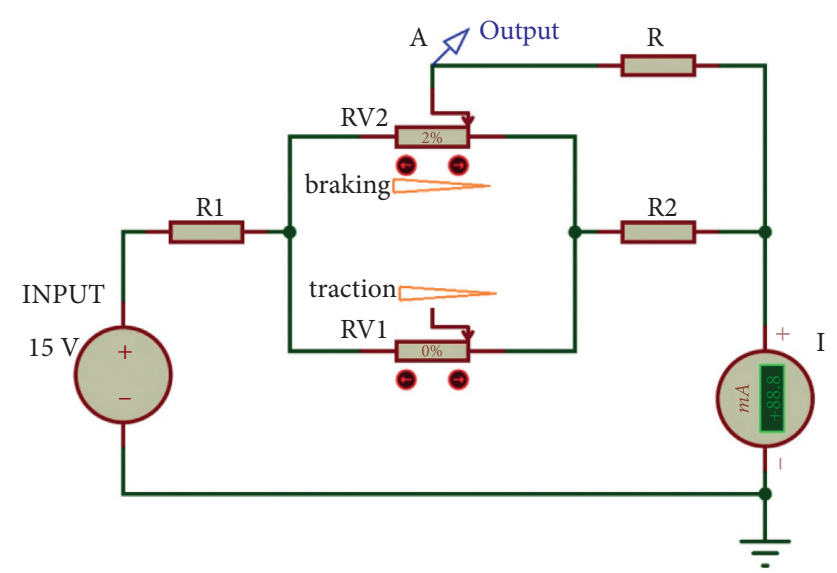

Figure 3: The circuit diagram of motorman controller potentiometer.

potentiometer is changed, thereby issuing instructions to adjust the speed of the locomotive. In this paper, 101 kinds of data in the potentiometer from the " 0 " slide to the braking process are randomly injected into each fault.

Fault description is shown in Table 3.

In the Proteus simulation software, each class of 101 sets of fault samples (505 groups) is obtained. The fault samples are divided into 2 categories according to the category. The first one is the training sample (group 340), and the second one is the test sample (group 165).

4.2. Feature Reduction. The driver controller potentiometer has a lot of fault types and fault element prerequisite attributes, and the circuit has some branches and trunk roads, such as voltage, current, resistance of the branches and trunk roads, sliding ratio of sliding rheostat in braking process $R V 2$, and so on. The correct choice of characteristic quantity is not only easy to diagnose but also simplifies the learning model. Because the potentiometer is mainly operated by regulating the resistance variation of the change of voltage to control the speed of railway vehicles, this paper mainly
TABLE 3: Fault description of potentiometer.

\begin{tabular}{lccc}
\hline Number & Fault description & Nominal value & Fault value \\
\hline$D_{0}$ & Fault-free & & \\
$D_{1}$ & RV2 $\uparrow$ & $1043 \Omega$ & {$[1460.2,2086] \Omega$} \\
$D_{2}$ & RV2 $\downarrow$ & $1043 \Omega$ & {$[104.3,625.8] \Omega$} \\
$D_{3}$ & GRB $($ R5) & & {$[10,1000] \Omega$} \\
$D_{4}$ & ROP $($ RV2) & $1043 \Omega$ & {$[100,100000] K \Omega$} \\
\hline
\end{tabular}

assumes the circuit output voltage, main circuit current, and output resistance as characteristic quantities. For more complex circuit or circuit having a plurality of output terminals, more characteristic quantities can be chosen.

Using the training data, the number of samples is $T=$ 340 and the fault feature number is $P=3$. It is necessary to perform standardized treatment of original variables, that is, the original variables need to be standardized.

The mean is $x_{j}=\left[\begin{array}{lll}5.89 & 10.48 & 0.63\end{array}\right]$; the standard deviation is $S_{i j}=\left[\begin{array}{lll}2.68 & 2.23 & 0.40\end{array}\right]$; and the characteristic quantity is $\lambda_{j}=\left[\begin{array}{lll}2.672 & 0.306 & 0.022\end{array}\right]$. Calculate the contribution to the total variance of the principal components in the $i$ th rate, and the variance contribution rate is

$$
\operatorname{per}_{i}=\frac{\lambda_{j}}{\sum_{j=1}^{P} \lambda_{j} \times 100 \%=\left[\begin{array}{lll}
89.07 \% & 10.19 \% & 0.74 \%
\end{array}\right]}
$$

It shows that there are 2 total contribution rates of more than $95 \%$ principal components (because the characteristic of greater than 1 is only one factor by using SPSS analysis, it cannot be rotated, and two principal components are extracted by the gravel method); according to varimax rotation, the load matrix is obtained, and the correlation between the principal components and key fault feature is shown in Table 4.

The main elements usually take the total contribution rate of more than $95 \%$, which contain most of the original variable information. Through principal component analysis, two principal components of total contribution rate are more than $95 \%$, and output voltage coefficient of component 
TABLE 4: The correlation between the two principal components and the fault characteristics.

\begin{tabular}{lcc}
\hline Feature & Component 1 & Component 2 \\
\hline Output voltage & 0.924 & 0.379 \\
Electric current & -0.414 & 0.910 \\
Resistance & 0.855 & 0.495 \\
\hline
\end{tabular}

1 which is obtained by maximum variance rotation method is greater than 0.9 , the main current coefficient $(0.910)$ of component 2 is greater than 0.9 , and the two features are selected as an input of the BRB expert system. The number of rules and model structure are simplified, which can greatly improve the learning rate.

4.3. RIMER Algorithm. The output voltage is selected by 6 reference values: zero (Z), very small (VS), small (S), medium $(\mathrm{M})$, large $(\mathrm{L})$, and very large $(\mathrm{VL})$, that is, $A_{1} \in\{Z, V S, S, M, L, V L\}$. The electric current is selected by 7 reference values: very small (VS), medium small (MS), small $(\mathrm{S})$, medium $(\mathrm{M})$, large $(\mathrm{L})$, medium large $(\mathrm{ML})$, and very large (VL), $A_{2} \in\{V S, M S, S, M, L, M L, V L\}$. The semantic value and referenced values of features are shown in Tables 5 and 6.

The potentiometer has five fault states which are normal work (D0), element parameter positive deviation (D1), element parameter negative deviation (D2), external load (D3), and potentiometer open circuit (D4), D = [D0, D1, D2, D3, D4]. Above the semantic value, it needs to be quantified, and the referenced values of the fault states are shown in Table 7.

\subsubsection{The Initial BRB Potentiometer Fault Diagnosis.} Because the output voltage has 6 reference values and the current has 7 reference values, there are 42 belief rules in belief rule base (BRB). Divide the experimental data into 152 sets of training data and 238 sets of test data $\left(\begin{array}{ll}x_{152,1} & x_{152,2}\end{array}\right)=\left(\begin{array}{ll}4.27253 & 11.4\end{array}\right), \quad\left(\begin{array}{ll}x_{283,1} & x_{283,2}\end{array}\right)=$ ( 3.4379311 .9 ). The output voltage and electric current reference values of the two sets data are very similar, and the semantic value $A_{1}$ is between $S$ and $M$. The semantic value $A_{2}$ is between $M$ and $L$. However, 152 groups of fault data are D2 (component parameters $R V 2 \downarrow$ ), and the reference value is 3; 238 groups of fault data are $\mathrm{D}_{3}$ (external load failure), and the reference value is 4 , which is diagnosed by the initial confidence rule base.

(1) The initial BRB diagnosis processes of 152 data $\left(\begin{array}{ll}x_{152,1} & x_{152,2}\end{array}\right)=\left(\begin{array}{ll}4.27523 & 11.4\end{array}\right)$ are shown in Tables 8-10.

$$
\begin{aligned}
\left\{\left(D_{0}, \beta_{1, k}\right), \ldots\left(D_{N}, \beta_{N, k}\right)\right\}= & \left\{\left(D_{0}, 0.0320\right),\right. \\
& \left(D_{1}, 0\right),\left(D_{2}, 0.650\right), \\
& \left.\left(D_{3}, 0.3180\right),\left(D_{4}, 0\right)\right\} .
\end{aligned}
$$

That is, the probability output of the fault sample belongs to the fault types; the probability of the third fault is the largest.
TABLE 5: The referenced values of $A_{1}$.

\begin{tabular}{lcccccc}
\hline Semantic values & Z & VS & S & M & L & VL \\
\hline Referenced values & 0 & 2.4 & 3 & 6 & 8 & 10.1 \\
\hline
\end{tabular}

TABLE 6: The referenced values of $A_{2}$.

\begin{tabular}{lccccccc}
\hline Semantic values & VS & MS & S & M & L & ML & VL \\
\hline Referenced values & 7.5 & 9 & 10 & 11.5 & 13.5 & 14.5 & 21.5 \\
\hline
\end{tabular}

TABLE 7: The referenced values of the fault states.

\begin{tabular}{lccccc}
\hline Semantic values & D0 & D1 & D2 & D3 & D4 \\
\hline Referenced values & 1 & 2 & 3 & 4 & 5 \\
\hline
\end{tabular}

Table 8: Relative membership degree of $A_{1}$.

\begin{tabular}{lcccccc}
\hline Semantic values & Z & VS & S & M & L & VL \\
\hline Membership & 0 & 0 & 0.575823 & 0.424177 & 0 & 0 \\
\hline
\end{tabular}

Confidence relative evaluation results of ER algorithm:

$$
\begin{aligned}
y_{i} & =\sum_{1}^{N} D_{n} \beta_{n}^{k} \\
& =3.254 .
\end{aligned}
$$

Fault status belongs to D3, component parameters $R V 2 \downarrow$. The diagnosis is correct.

(2) The initial BRB diagnosis processes of 238 data $\left(\begin{array}{ll}x_{238,1} & x_{238,2}\end{array}\right)=\left(\begin{array}{ll}3.43793 & 11.9\end{array}\right)$ are shown in Tables 11-13.

$$
\begin{gathered}
\left\{\left(D_{0}, \beta_{1, k}\right), \ldots\left(D_{N}, \beta_{N, k}\right)\right\}=\left\{\left(D_{0}, 0\right),\left(D_{1}, 0\right),\right. \\
\left.\left(D_{2}, 0.5558\right),\left(D_{3}, 0.4442\right),\left(D_{4}, 0\right)\right\} .
\end{gathered}
$$

That is, the probability output of the fault sample belongs to the fault types; the probability of the third fault is the largest, and it is inconsistent with the real situation.

Confidence relative evaluation results of ER algorithm:

$$
\begin{aligned}
y_{i} & =\sum_{1}^{N} D_{n} \beta_{n}^{k} \\
& =3.4442 .
\end{aligned}
$$

Fault status belongs to D3, component parameters $R V 2 \downarrow$. The diagnosis is wrong. In the next section, the initial $\mathrm{BRB}$ parameters are optimized to improve the accuracy.

4.4. The Fault Diagnosis Using the Optimized BRB. The BRB parameters $V=\left[\theta^{1} \cdots \theta^{K} \cdots \delta^{1} \cdots \delta^{m} \cdots \beta_{1}^{k} \cdots \beta_{n}^{k}\right]^{T}$ are optimized by establishing the true reference value of the data category and the variance of the actual output value. It is diagnosed by the belief rule base after optimization. 
TABLE 9: Relative membership degree of $A_{2}$.

\begin{tabular}{lccccccc}
\hline Semantic values & Z & VS & S & M & L & VL & VL \\
\hline Membership & 0 & 0 & 0 & 0.067 & 0.933 & 0 & 0 \\
\hline
\end{tabular}

TABLE 10: The activation weight calculation section of the $k$ th rule.

\begin{tabular}{lccccc}
\hline Article $k$ these rules & 17 & 18 & 24 & 25 & Other rules \\
\hline$\omega_{k}$ & 0.03839 & 0.53744 & 0.02828 & 0.39589 & 0 \\
\hline
\end{tabular}

ER algorithm is used to calculate the relative evaluation results of confidence.

TABLE 11: Relative membership degree of $A_{1}$.

\begin{tabular}{lcccccc}
\hline Semantic values & Z & VS & S & M & L & VL \\
\hline Membership & 0 & 0 & 0.8540233 & 0.1459767 & 0 & 0 \\
\hline
\end{tabular}

TABLe 12: Relative membership degree of $A_{2}$.

\begin{tabular}{lccccccc}
\hline Semantic values & VS & MS & S & M & L & ML & VL \\
\hline Membership & 0 & 0 & 0 & 0.8 & 0.2 & 0 & 0 \\
\hline
\end{tabular}

TABLE 13: Kth rule activation weight calculation.

\begin{tabular}{lccccc}
\hline Article $k$ these rules & 18 & 19 & 25 & 26 & Other rules \\
\hline$\omega_{k}$ & 0.6832 & 0.5723 & 0.1168 & 0.0292 & 0 \\
\hline
\end{tabular}

ER algorithm is used to calculate the relative evaluation results of confidence.

(1) The optimized BRB diagnosis process of 152 data. ER algorithm is used to calculate the relative evaluation results of evaluation.

$$
\begin{aligned}
\left\{\left(D_{0}, \beta_{1, k}\right), \ldots\left(D_{N}, \beta_{N, k}\right)\right\}= & \left\{\left(D_{0}, 0.0739\right),\left(D_{1}, 0.2242\right),\right. \\
& \left(D_{2}, 0.4886\right),\left(D_{3}, 0.0195\right), \\
& \left.\left(D_{4}, 0.1889\right)\right\} .
\end{aligned}
$$

That is, the probability output of the fault sample belongs to the fault types, and the probability of the third fault is the largest.

The evaluation results of semantic value.

$$
\begin{aligned}
y_{i} & =\sum_{1}^{N} D_{n} \beta_{n}^{k} \\
& =3.0106 .
\end{aligned}
$$

Fault status belongs to D3, component parameters $R V 2 \downarrow$. The diagnosis is correct and more accurate than the original BRB.

(2) The optimized BRB diagnosis process of 238 data. ER algorithm is used to calculate the relative evaluation results of evaluation.

$$
\begin{aligned}
\left\{\left(D_{0}, \beta_{1, k}\right), \ldots\left(D_{N}, \beta_{N, k}\right)\right\}=\left\{\left(D_{0}, 0.0055\right),\left(D_{1}, 0.0263\right),\right. & \\
& \left(D_{2}, 0.4440\right),\left(D_{3}, 0.1365\right), \\
& \left.\left(D_{4}, 0.3761\right)\right\} .
\end{aligned}
$$

That is, the probability of the output result sample belongs to the fault category, and the probability of third kinds of faults is the largest, which is not consistent with the real situation.

The evaluation results of semantic value.

$$
\begin{aligned}
y_{i} & =\sum_{1}^{N} D_{n} \beta_{n}^{k} \\
& =3.8166 .
\end{aligned}
$$

It is worth noting here that it belongs to D4 fault when $3.6 \leq y_{i}<4.4$.

Fault state belongs to D4, external load fault. The diagnosis is correct. Through the comparison of the above two methods, in terms of precision or accuracy, the updated BRB diagnosis effect is much better than the initial BRB.

The fault category of the testing data can be calculated by the same way. Figure 4 shows the output of the 


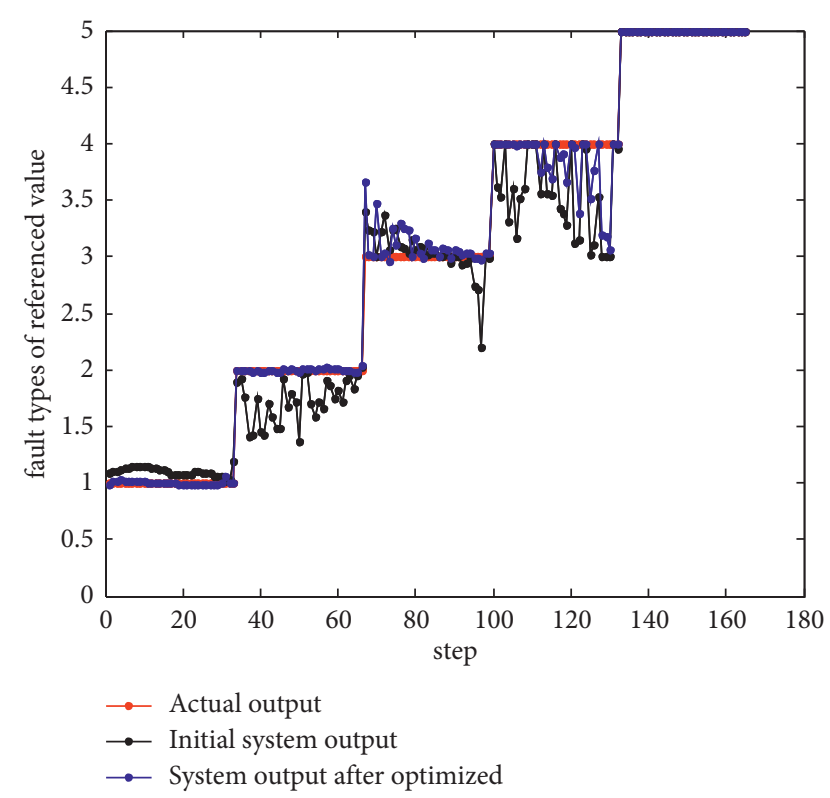

Figure 4: Comparison chart of optimized system fault diagnosis results.

potentiometer's fault state using initial and optimized BRB. Figure 5 shows the fault feature and output of the potentiometer's fault state using initial and optimized BRB.

As can be seen from Figure 5, the fault type coordinates of the optimized BRB diagnosis system are more suitable to the actual reference value; as can be seen from Figure 4, the reference value of the optimized BRB fault diagnosis system is similar to the actual reference value. The blue line in the figure is almost entirely within the range of the true reference value; therefore, the updated diagnostic results are more accurate and the diagnostic accuracy is higher.

\subsection{Fault Diagnosis of Optimized BRB Potentiometer Based on} Unnormalized Activation Weight. The comparative study is also done by the ER algorithm of nonstandardized $\omega_{k}$ using the same training and testing data. Figure 6 shows the comparison chart of output value of unnormalized activated weights fault diagnosis and true reference value.

ER algorithm of unnormalized is

$$
\omega_{k}=\theta_{k} \prod_{i=1}^{M}\left(\alpha_{i}^{k}\right) \bar{\delta}_{i} .
$$

The accuracy by using unnormalized activated weights is more than that using initial BRB. However, the accuracy is less than that of optimized $\mathrm{BRB}$ of normalized activated weights. It proves that when there is a conflict of rules and the weight of conflict evidence is very large, it is suitable for fusion after normalization.

4.6. The Fault Diagnosis Using the PCA to Reconstruct Fault Feature in the Optimized BRB. The principal component of reconstruction by $\mathrm{PCA}$ is used as input feature of the $\mathrm{BRB}$ model, not the varimax rotation back stepping features. This

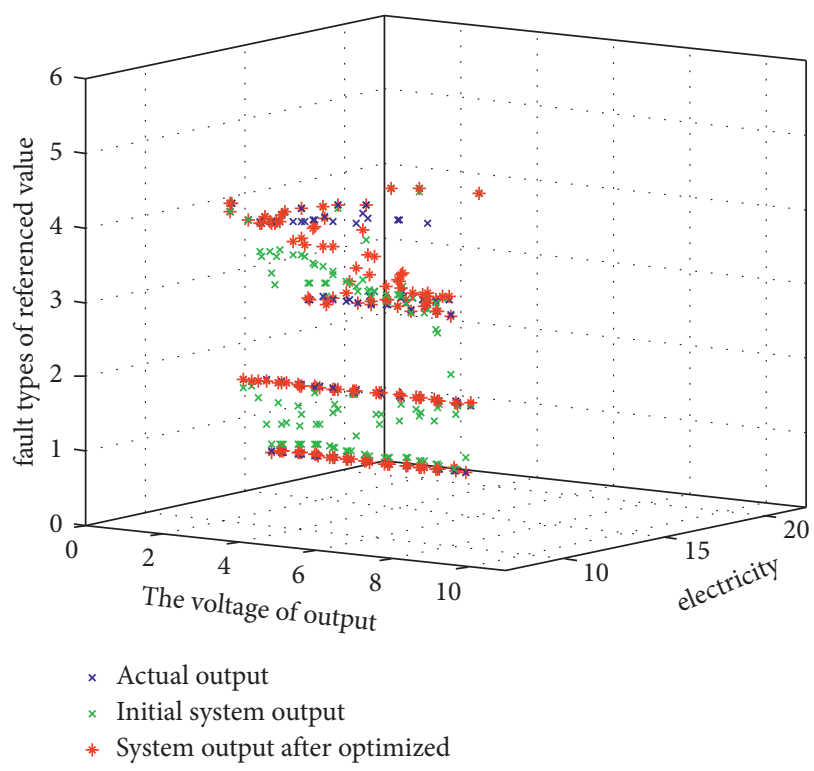

FIgURE 5: Three-dimensional diagram of optimized system fault diagnosis results comparison.

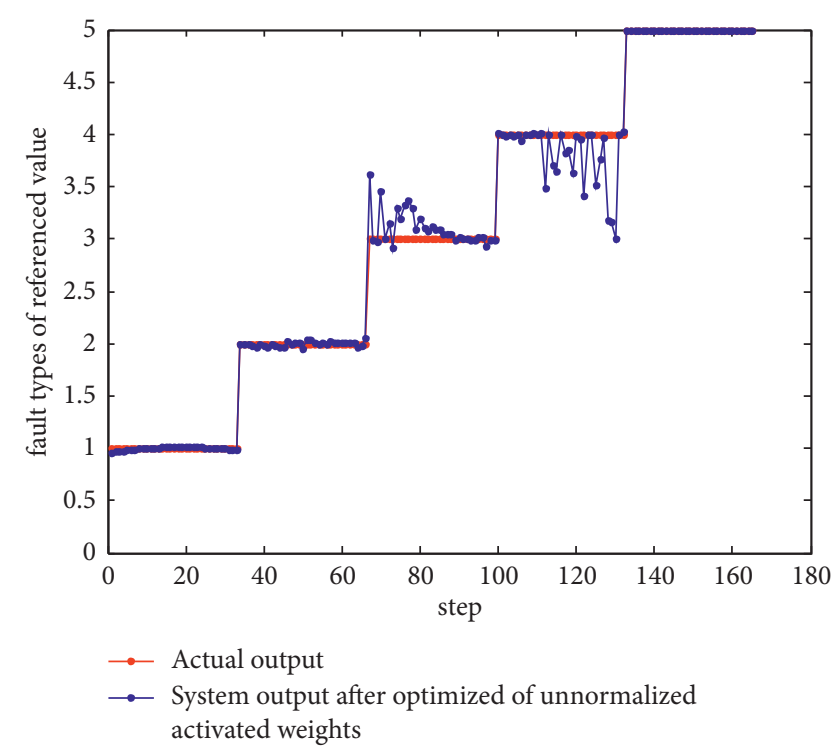

Figure 6: Comparison of the diagnostic results of the unnormalized activation weight potentiometer and the true value of the test data.

paper also conducts a comparative study of this method, as shown in Figure 7. The accuracy and error are less than those of optimized BRB of varimax rotation. The data diagnosis process without physical meaning and qualitative knowledge proved to be difficult.

4.7. The Fault Diagnosis Using the Neural Net Algorithm. In this section, the potentiometer is diagnosed by using BP neural network. The parameter settings are as follows: net.train Param.epochs $=340$ and net.train Param.goal $=1 e-5$. Others are the default values. The training data and testing data are the same text. Figure 8 


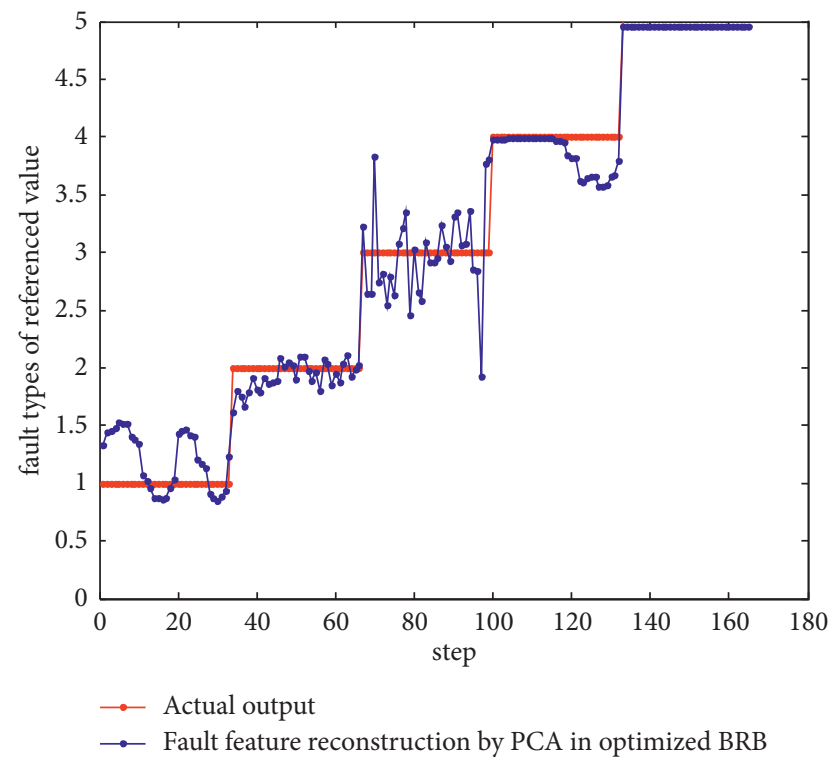

FIGURE 7: PCA reconstructed data are used to optimize the BRB potentiometer fault diagnosis result and the true value comparison chart of the test data.

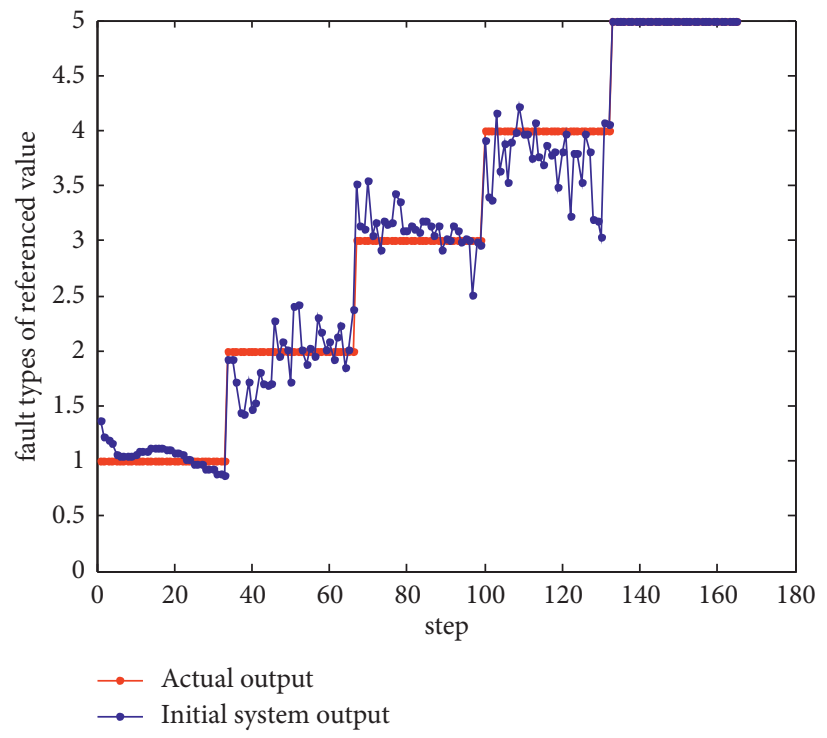

FIGURE 8: Comparison chart of actual output and initial system output.

TABLE 14: Comparison of the results of different methods.

\begin{tabular}{lcr}
\hline Method & Accuracy (\%) & MSE \\
\hline Optimized BRB & 95.8 & 0.1603 \\
Initial BRB & 83.6 & 0.3091 \\
ER algorithm of unnormalized $\omega_{k}$ in optimized BRB & 95.2 & 0.1722 \\
Fault feature reconstruction by PCA in optimized BRB & 87.9 & 0.2545 \\
BP neural network & 89.1 & 0.2396 \\
\hline
\end{tabular}

shows the comparison chart of output value of BP neural network fault diagnosis and true reference value.

Moreover, BP neural network belongs to the "black box" method, so it is difficult to explain the complicated relationship of complex system between the input and output. Only the category label corresponding to the fault sample can be output, and other diagnostic information, such as the probability of each fault category in the fault sample, cannot be output.

4.8. Comparison of the Results of Different Methods. Two methods of true reference value and output reference value 
are used to evaluate the fault diagnosis capability, as shown in Table 14. The results prove that the optimized BRB results are better than the other two methods, with better accuracy and lower error.

\section{Conclusion}

Taking the controller potentiometer as an example, a fault diagnosis model is established in this paper. This paper focuses on the fault diagnosis methods of railway passenger cars, which can also be developed into a fault diagnosis system of railway passenger cars. The feasibility study of applying the PCA-BRB fault diagnosis method to electronic circuit of the railway vehicle is introduced. Firstly, the limited fault feature acquisition and simplified circuit model were solved by the principal component analysis and maximum variance rotation method. In this method, qualitative knowledge and quantitative information are combined effectively, and two fusion methods of normalized activation weights and nonnormalized activation weights are proposed according to different actual situations. Finally, the initial BRB parameters are optimized. Compared with initial $B R B$, unnormalized activation weights in optimized BRB, PCA, and neural network for reconstruction of fault features in optimized BRB, the proposed method has higher diagnostic accuracy and minimum error. The fault diagnosis method proposed in this paper can find the faults of railway vehicles and ensure the reliability of railway operation.

Further efforts should be made to further study the following aspects in the future:

(1) Higher precision optimization algorithm could be explored to improve the optimization ability of confidence rule base model.

(2) Although this paper only takes the common circuit faults as an example, more emphasis will be placed on the study of fault diagnosis methods for rail cars in the future, which can be developed into the fault diagnosis system for rail cars.

\section{Data Availability}

The data used to support the findings of this study are currently under embargo while the research findings are commercialized. Requests for data, [6/12 months] after publication of this article, will be considered by the corresponding author.

\section{Conflicts of Interest}

The authors declare that they have no conflicts of interest.

\section{Acknowledgments}

This research was supported by the Science and Technology Project of the 13th Five-Year Plan of Education Department of Jilin Province (no. JJKH20200655KJ) and the Science and Technology Development Project of Jilin Province (no. 20200301038RQ).

\section{References}

[1] B. S. Kariyappa, "A survey on fault diagnosis of analog circuits: taxonomy and state of the art," AEUE-International Journal of Electronics and Communications, vol. 73, pp. 68-83, 2017.

[2] H. Yuan, G. Chen, and Y. Xie, "Feature evaluation and extraction based on neural network in analog circuit fault diagnosis," Journal of Systems Engineering and Electronics, vol. 18, no. 2, pp. 434-436, 2007.

[3] G. Song, S. Jiang, and H. Wang, "Analog circuit fault diagnosis approach using optimized SVMs based on MST algorithm," IEEE, vol. 4, pp. 236-240, 2011.

[4] A. Zhang, C. Chen, and B. Jiang, "Analog circuit fault diagnosis based UCISVM," Neurocomputing, vol. 173, pp. 1752-1760, 2016.

[5] L. Oukhellou, A. Debiolles, and T. Denœux, "Fault diagnosis in railway track circuits using Dempster-Shafer classifier fusion," Engineering Applications of Artificial Intelligence, vol. 23, no. 1, pp. 117-128, 2010.

[6] D. R. Huang, L. Y. Ke, X. Y. Chu, L. Zhao, and B. Mi, “Fault diagnosis for the motor drive system of urban transit based on improved Hidden Markov Model," Microeletronics Reliability, vol. 82, pp. 179-189, 2018.

[7] X. T. Li, Research on Fault Diagnosis System of Locomotive Electric Drive Circuit, Northwestern Polytechnical University, Xi'an, China, 2003.

[8] J. B. Yang, J. Liu, and J. Wang, "Belief rule-base inference methodology using the evidential reasoning approachRIMER," IEEE Transactions on Systems, vol. 36, no. 2, pp. 266-285, 2006.

[9] W. Zhang, C. Shi, C. H. Hu, and Z. Z. Zhou, Fault Diagnosis of Engine Based on RIMER Expert System, System Simulation Technology, China, 2011.

[10] Z. W. Liu, "Research on fault diagnosis method of locomotive wheelset based on neural network," Application of Automation, vol. 8, pp. 17-18, 2019.

[11] Z. Tong and Y. P. Zhang, "Research on locomotive wheelset fault diagnosis system based on BP neural network," Railway Technology Innovation, vol. 109, no. 1, pp. 21-23, 2009.

[12] L. L. Chang, L. I. Meng-Jun, and L. U. Yan-Jing, "Structure learning for belief rule base using principal component analysis," Systems Engineering-Theory \& Practice, vol. 34, no. 5, pp. 1297-1304, 2014.

[13] Z. J. Zhou, Belief Rule Base of Expert System and Complex System Modeling, Science Press, China, 2011.

[14] G. Shafer, "A mathematical theory of evidence," Technometrics, vol. 20, no. 1, p. 242, 1976.

[15] Z. Zhou, X. B. Xu, and C. L. Wen, "An optimal method for combining conflicting evidences," Acta Automatica Sinica, vol. 38, no. 6, p. 976, 2012.

[16] J. B. Yang and D. L. Xu, "Evidential reasoning rule for evidence combination," Artificial Intelligence, vol. 205, no. 205, pp. 1-29, 2013.

[17] J. M. Bradley and E. M. Atkins, "A cyber-physical optimization approach to mission success for unmanned aircraft systems," Journal of Aerospace Computing, Information, and Communication, vol. 11, no. 1, pp. 48-60, 2014.

[18] G. Li, Z. Zhou, and C. Hu, "A new safety assessment model for complex system based on the conditional generalized minimum variance and the belief rule base," Safety Science, vol. 93, pp. 108-120, 2017. 
[19] X. Tang and A. Xu, "Practical analog circuit diagnosis based on fault features with minimum ambiguities," Journal of Electronic Testing, vol. 32, no. 1, pp. 83-95, 2016.

[20] X. Tang, X. U. Aiqiang, and L. I. Wenhai, A Stochastic Mutant Generation Method for Multi-Level Analog Circuits, China Measurement \& Test, Shanghai, China, 2016.

[21] L. Wang, The Research of Master Controller for Metro, Dalian University of Technology, Dalian, China, 2015.

[22] W. S. Pritchard, "PCAVR: a portable laboratory program for performing varimax-rotated principal components analysis of event-related potentials," Brain Research Bulletin, vol. 13, no. 3, pp. 465-473, 1984.

[23] Y. Xiao and L. Feng, "A novel linear ridgelet network approach for analog fault diagnosis using wavelet-based fractal analysis and kernel PCA as preprocessors," Measurement, vol. 45, no. 3, pp. 297-310, 2021. 